\title{
QUALIDADE DE VIDA RELACIONADA À SAÚDE (HRQL) DE ADULTOS ENTRE 50 E 80 ANOS PRATICANTES DE ATIVIDADE FISICA REGULAR: APLICAÇÃO DO SF-36
}

Andréa Kruger Gonçalves

Adriane Ribeiro Teixeira²

Cíntia de la Rocha Freitas ${ }^{3}$

Eliane Jost Blessmann ${ }^{4}$

Laysla Roedel ${ }^{5}$

Mariane Goettert Martins 5

Eduardo Hauser ${ }^{5,8}$

Eliane Mattana Griebler 5,8

Natalia Schardosim Copetti

Ângelo José Gonçalves Bós ${ }^{7}$

1 Profa. Dra. em Educação Física/UFRGS, coordenação CELARI/UFRGS. E-mail: andreakg@terra. com.br

2 Profa. Dra. em Fonoaudiologia/UFRGS. E-mail: adriteixeira@yahoo.com.br

3 Profa. Dra. em Educação Física/UFSC. E-mail: cintiadelarocha@gmail.com

4 Técnica Assistente Social Ms./UFRGS, coordenação CELARI/UFRGS. E-mail: eliane.blessmann@ ufrgs.br

5 Acadêmicos de Educação Física/UFRGS e estagiários bolsistas CELARI/UFRGS. E-mail: celari@ ufrgs.br

6 Acadêmica do Curso de Fonoaudiologia da UFRGS - Bolsista PROBIC FAPERGS UFRGS.

7 Médico, Professor do Programa de Pós-Graduação em Gerontologia Biomédica da Pontifícia Universidade Católica do Rio Grande do Sul.E-mail: angelo.bos@pucrs.br

8 Bolsistas de iniciação científica PROBIC FAPERGS UFRGS. 
resumo

A qualidade de vida relacionada à saúde-HRQL possui um caráter multidimensional com integração da saúde física, do bem-estar psicológico e da satisfação social. O objetivo foi avaliar a qualidade de vida relacionada à saúde (HRQL) de participantes de meia-idade e de idosos de um projeto de atividade física regular. $\bigcirc$ tipo de estudo foi descritivo, com corte transversal, sendo que a amostra foi composta por 40 adultos com idade entre 50 e 80 anos, integrantes do CELARI da ESEF/UFRGS. O instrumento utilizado foi o SF-36 e a análise empregada utilizou a estatística descritiva e o teste de correlação de Pearson. A pontuação dos domínios por ordem decrescente foi: AS, AF, AE, EGS, CF, SM, VIT, DOR. Houve correlação positiva significativa entre a maioria dos domínios do SF-36. As correlações não significativas ocorreram entre EGS x AS, SM x AF. A idade apenas correlacionou-se com o domínio EGS. A HRQL da amostra mostrou-se com valores superiores, ao ser comparada com a de outros estudos. Os valores atingiram no mínimo 70\% de pontuação, na média dos domínios. Os resultados indicaram um nível satisfatório de HRQL e relação entre os domínios do SF-36.

palavras - chave

Envelhecimento. Qualidade de Vida. Atividade Física. Saúde.

\section{$1 \quad$ Introdução}

A ação de envelhecer é considerada um processo universal, dinâmico e irreversível. Para Spirduso (2005), os seres humanos envelhecem de forma diferente entre e si e em ritmos diversos, sendo que as opções ao longo da vida influenciam a qualidade e o tempo de vida. A morte de pessoas idosas, na maioria das vezes, é causada como decorrência de um conjunto de mudanças fisiológicas e crônicas, e não simplesmente devido à idade. Envelhecer varia de acordo com inúmeros fatores biopsicossociais, os quais variam, de características genéticas às diferenças educacionais e culturais. Para Gonçalves e Groenwald (2005), o processo de envelhecimento é diferente, de acordo com o estilo de vida adotado, sendo que o crescente aumento da expectativa de vida da população demanda a necessidade de medidas efetivas, para garantir qualidade aos anos obtidos, com melhores condições de vida. 
A esperança de vida, que era de 43,3 anos na década de 1950 passou para 72,5 anos em 2007, projetando-se que no ano de 2025, atinja 80 anos (IBGE apud FIEDLER; PERES, 2008). A expressão qualidade de vida tem sido cada vez mais utilizada quando o tema é envelhecimento, devido à preocupação de proporcionar condições de vida adequadas e satisfatórias para a população, que tem tido, especialmente nas últimas décadas, um aumento expressivo da expectativa de vida. Contudo, existe carência de estudos e políticas de atenção à saúde do idoso.

Um dos fatores que tem sido associado com melhora da qualidade de vida é a prática de atividade física regular, especialmente pela sua importância para a manutenção da independência nas atividades cotidianas do diadia. Entre as características do envelhecimento, aponta-se que ocorre diminuição de capacidades motoras (resistência, força, flexibilidade, equilíbrio), bem como, aumento da probabilidade de doenças. Nos aspectos psicossociais destacam-se a perda de entes queridos, aposentadoria, saída dos filhos de casa, diminuição do convívio social (GONÇALVES, 1999). Todo esse conjunto de características é influenciado pela genética e estilo de vida adotado: alimentação, atividade física, sono, entre outros (PU; NELSON, 2001).

Um dos termos utilizados relacionados à avaliação dos efeitos da atividade física na vida das pessoas tem sido a qualidade de vida. De acordo com Pimenta et al. (2008), o conceito de qualidade vida confunde-se com estilo de vida, condições de vida e situações de vida, não tendo, apesar de discussão no mundo inteiro, uma uniformidade. Para os autores anteriores é preciso diferenciar qualidade de vida, de estado de saúde, de acordo com três dimensões principais: saúde mental, função física e função social. Sendo que, para saúde o primordial é a segunda dimensão, enquanto que para a qualidade de vida, incluem-se as três dimensões citadas.

O termo qualidade de vida incorpora tanto questões de condições e estilos de vida, como desenvolvimento sustentável e direitos humanos e sociais, abordando ainda a subjetividade e o seu caráter de múltiplas dimensões. Para Sousa, Galante e Figueiredo (2003), qualidade de vida inclui diferentes dimensões da vida, sendo que os modelos que a avaliam incluem desde a satisfação com a vida, bem-estar social, até modelos baseados em conceitos de independência, controle, competências sociais e cognitivas. As autoras reforçam o fato de que, ao avaliar a qualidade de vida na velhice, é preciso estar atento à questão da dependência, enquanto decorrência das alterações do processo do envelhecimento, ou como consequência do ambiente nãoadaptado. Do mesmo modo, é preciso também considerar os processos de 
senescência e de senilidade, não associando a ocorrência de patologias como algo pontual e factual na vida de pessoas idosas.

De acordo com Fleck (2008), o termo "qualidade vida relacionada à saúde" (Health Related Quality of Life-HRQL) é utilizado numa perspectiva funcionalista. Deste modo, se a saúde estiver influenciada negativamente, afetará a independência do idoso, resultando em menor qualidade de vida. Ressalta-se o fato de que esse processo não é linear, porém, com influências mútuas. Mota et al. (2006) destaca que a informação sobre a qualidade de vida relacionada à saúde (HRQL) complementa os dados de morbidade e mortalidade. A HRQL possui um caráter multidimensional com integração da saúde física, do bem-estar psicológico e da satisfação social.

Para Navega e Oishi (2007) existem problemas clínicos e sociais que podem influenciar de modo negativo as atividades do cotidiano e, assim, afetar o bem-estar e a qualidade de vida relacionada à saúde. Para os autores, a presença de comorbidades (as quais tendem a aumentar com os anos) também afetam a HRQL. Os autores indicam que a prática regular de atividade física pode melhorar a qualidade de vida das pessoas, inclusive daquelas com doenças crônico-degenerativas. Segundo Sguizzatto, Garcez-Leme e Casimiro (2006), a atividade física regular tem sido vista como um dos procedimentos mais efetivos para promover a qualidade de vida na população.

O objetivo deste estudo foi avaliar a qualidade de vida relacionada à saúde (HRQL) de participantes de meia-idade e idosos de um projeto de atividade física regular.

\section{Metodologia}

O estudo foi do tipo descritivo, com corte transversal. A amostra foi, por conveniência, composta por voluntários, perfazendo 40 pessoas com idades entre 50 e 80 anos: 34 mulheres e 6 homens, todos praticantes de atividade física regular no projeto de extensão universitária "Centro de Esporte, Lazer e Recreação do Idoso"/CELARI da Escola de Educação Física da Universidade Federal do Rio Grande do Sul/UFRGS. Os critérios para inclusão na pesquisa foram: ter 50 anos de idade, ou mais, ser alfabetizado, ser independente funcionalmente e ser integrante do projeto de extensão há, no mínimo, seis meses.

O instrumento utilizado foi o "The Medical Outcomes Study 36 - Item Short Form Health Survery-Short Form-36" (SF-36) e um questionário com dados informativos quanto ao sexo e idade. O SF-36 é um dos instrumentos 
mais utilizados no mundo para avaliação da qualidade de vida e foi desenvolvido no final dos anos 80 nos Estados Unidos. No Brasil, foi traduzido e validado (CICONELLI, 1997), mostrando-se adequado às condições socioeconômicas e culturais da população brasileira. O SF-36 é um questionário multidimensional, formado por 36 itens, englobados em 8 componentes, ou domínios: capacidade funcional, aspectos físicos, dor, estado geral da saúde, vitalidade, aspectos sociais, aspectos emocionais e saúde mental. Apresenta um escore final de 0 a 100, no qual zero corresponde ao pior estado geral e 100 ao melhor estado de saúde (LEMOS et al., 2006). Deste modo, esse instrumento mede a qualidade de vida, de acordo com a saúde relatada.

A análise do SF-36 é realizada a partir da soma das questões e agrupada de acordo com os domínios, para após, calcular o Raw-scale, que considera o valor obtido, o valor mínimo e a variação de cada um dos domínios multiplicados por 100, finalizando com o mesmo peso para todos os domínios. Cada componente do SF-36 corresponde a um valor, que varia de zero a 100, onde zero corresponde ao pior e 100, à melhor qualidade de vida relacionada à saúde.

A pesquisa foi aprovada pelo Comitê de Ética do Instituto de Psicologia da UFRGS, conforme protocolo de número 2010036, e os sujeitos assinaram termo de consentimento livre e esclarecido.

Os dados foram analisados a partir da categorização dos componentes do SF-36, de acordo com o protocolo, para após, utilizar técnicas de estatística descritiva com cálculos de média e desvio padrão. O coeficiente de correlação de Pearson foi calculado para verificar a existência de correlação entre os domínios e componentes do SF-36, com nível de significância adotado de 5\% $(p \leq 0,05)$. Foi utilizado o programa estatístico SPSS 18.0.

As limitações desse estudo estão vinculadas à impossibilidade de generalização dos resultados, devido ao tipo de amostra utilizada. Outro fator adicional refere-se ao próprio instrumento, especialmente a motivação para participar do estudo, a qual pode interferir na escolha das respostas; bem como, a influência do grau de estudo, pela sua associação com a capacidade de entendimento das questões.

\section{Resultados e Discussão}

Inicialmente, apresenta-se a estatística descritiva dos componentes do SF-36, para, após, apresentar o teste de correlação de Pearson. 
Tabela 1 - Estatística descritiva dos componentes do SF-36 em adultos entre 50 e 80 anos praticantes de atividade física regular

\begin{tabular}{c|c|c|c|c|c}
\hline Componentes & N & Mínimo & Máximo & Média & Desv. pad. \\
\hline CF & 40 & 35 & 100 & 79,75 & 18,22 \\
AF & 40 & 25 & 100 & 85,00 & 25,19 \\
DOR & 40 & 22 & 100 & 68,30 & 21,96 \\
EGS & 40 & 32 & 100 & 80,65 & 17,20 \\
VIT & 40 & 40 & 100 & 70,50 & 18,46 \\
AS & 40 & 25 & 100 & 87,19 & 20,70 \\
AE & 40 & 0 & 100 & 81,66 & 32,87 \\
SM & 40 & 40 & 100 & 75,40 & 16,74 \\
\hline
\end{tabular}

Os componentes com maior pontuação no SF-36 na amostra estudada foram os AS-aspectos sociais, seguidos dos AF-aspectos físicos, AE-aspectos emocionais, EGS-estado geral de saúde, CF-capacidade funcional, SM-saúde mental, VIT-vitalidade e DOR-dor.

O componente $\mathrm{CF}$ questiona sobre as atividades que o avaliado poderia realizar atualmente, durante um dia comum, e se, devido à sua saúde, tem dificuldades para realizar determinadas atividades e o quanto de dificuldade apresenta para tal. O componente AF está diretamente associado ao impacto da saúde física no desempenho das atividades diárias, e ou profissionais, se houve limitações no período de quatro semanas. O componente DOR referese à dor no corpo que o paciente sentiu durante as últimas quatro semanas, $\mathrm{e}$ quanto essa dor interferiu no trabalho normal. Já o VIT, relaciona-se ao modo como a pessoa se sente, e como tudo tem acontecido durante as últimas semanas, em relação à sua disposição, considerando, tanto nível de energia, como de fadiga, para enfrentar novas tarefas. O AS está diretamente associado à percepção do sujeito a respeito de sua saúde física e de problemas emocionais e como esses afetam suas atividades sociais normais, em relação à família, aos vizinhos e aos amigos, ou nas atividades realizadas em grupo, durante as últimas quatro semanas. O AE relaciona-se ao reflexo das condições emocionais, ou problemas (sentir-se deprimido ou ansioso) no desempenho das atividades da vida diária, e ou profissionais. O SM associa-se à percepção do sujeito, a respeito de como se sentiu e como transcorre sua vida, no período de quatro semanas, em relação ao seu ânimo. Ele tem como função analisar a ansiedade, a depressão, as alterações de comportamento, ou des- 
controle emocional e o bem-estar psicológico. E, por último, o EGS refere-se à percepção subjetiva do estado geral de saúde do indivíduo.

O SF-36 avalia a qualidade de vida na perspectiva de saúde integral (não apenas ausência de doenças) e incorpora aspectos primordiais, como independência nas atividades diárias e relacionamento social. Os resultados permitem visualizar uma pontuação que tende a convergir entre os domínios, refletindo a interdependência entre os mesmos, de acordo com os pressupostos teóricos desta área de estudo. Por se tratar de um grupo com idade superior a 50 anos, alguns domínios indicaram menos pontuação, como a DOR (corroborado pelos domínios VIT e CF). Fato que pode estar associado com propensão a alguma doença (como osteoartrite), além da diminuição de força e flexibilidade, as quais dificultam a realização de atividades diárias, causando sobrecarga em algumas articulações, ou também, aumentando a exigência sobre o sistema muscular (o qual se encontra diminuído).

A maior pontuação foi no componente AS, e segundo lugar, no AF. Tais valores revelam as características da amostra de estudo: serem mais idosos e ativos. O processo de envelhecimento é caracterizado, socialmente, pela diminuição de relações sociais em função da aposentadoria, da viuvez e do "ninho vazio" (NERI, 1995; GONÇALVES, 1999). Deste modo, a inserção num grupo, como é o caso desta amostra, é algo muito valorizado. Sentir-se parte de algo, além da família (que para muitos é restrita pela morte do cônjuge, distância dos filhos, ou pelo fato que muitos são solteiros), com encontros frequentes, é motivo de permanência no projeto de extensão. O domínio AF indica que o desempenho nas atividades diárias não é afetado negativamente pela saúde física, parecendo estar associado com a prática de exercícios físicos, a qual é a opção adotada como estilo de vida. A manutenção, ou recuperação de capacidades físicas é viabilizada com a prática de atividade física regular, influenciando a saúde (PUGGARD, 2003; REBELATTO et al., 2006; PETERSON et al., 2009; BERLEZI et al.,2010). 


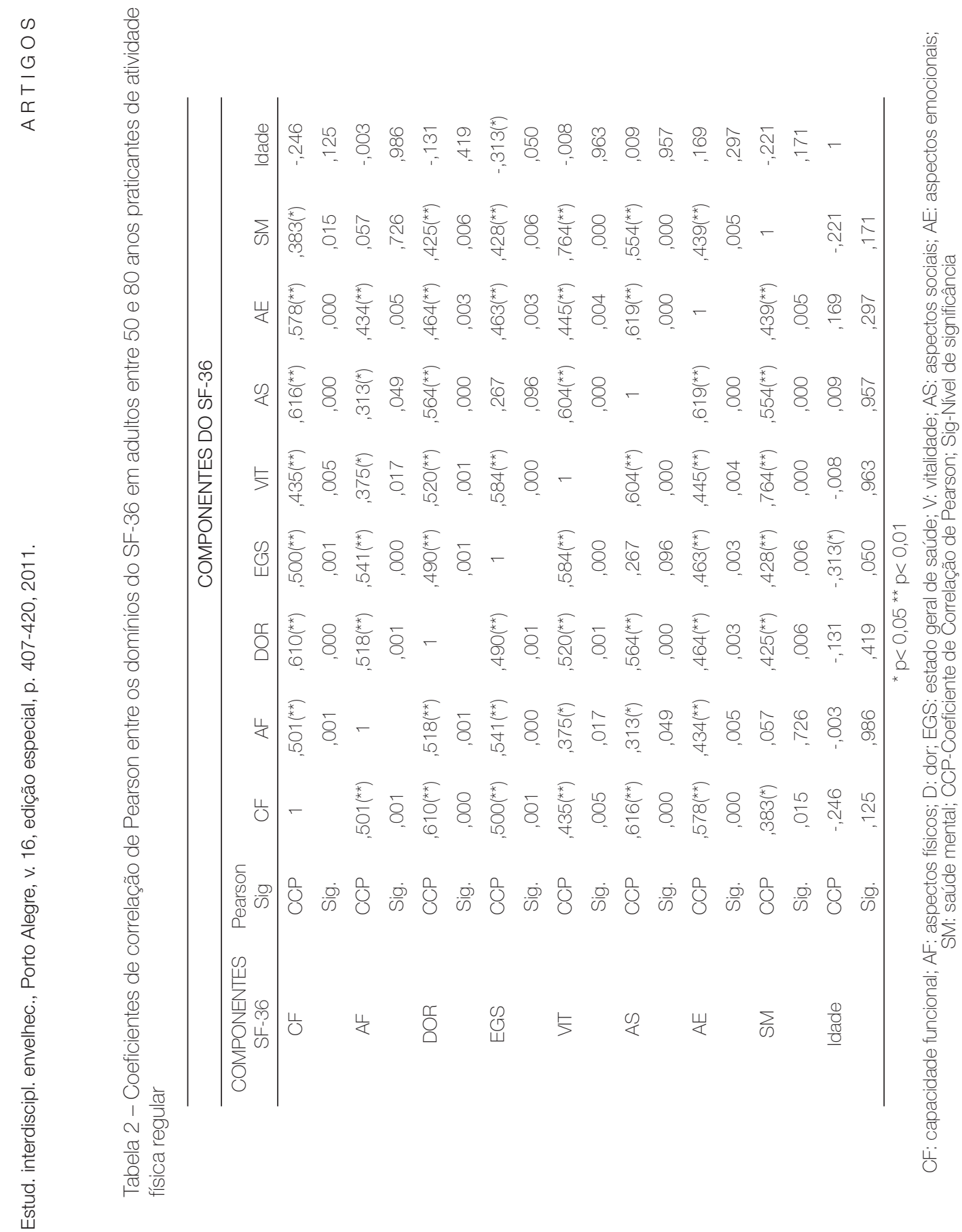


O teste de correlação de Pearson indica que houve relação positiva entre a maioria dos domínios do SF-36. As correlações que não foram significativas foram: EGS com AS, SM com AF. A idade apenas correlacionou-se com o domínio EGS, revelando que o número de anos de vida não interfere nos domínios da qualidade de vida, ao serem analisados de modo mais profundo.

O domínio EGS avalia a percepção subjetiva de saúde em duas questões gerais, ao contrário do restante do instrumento, em que cada um dos aspectos é aferido com mais especificidade. Deste modo, num primeiro momento, na amostra do estudo, quanto mais idade, melhor o EGS, porém, ao se questionar com mais profundidade cada faceta a idade não se relacionou de modo significativo. Tais resultados refletem a necessidade de mais estudos para observar a influência de diferentes hábitos e estilos de vida. A literatura aponta que a idade seria algo que interferiria de modo negativo (LIMA et al., 2009), porém, existem indícios de que mais do que os anos de vida, mas sim, as opções que são realizadas, é que interferem. No estudo de Castro et al. (2007) sobre qualidade de vida em idosos envolvidos em dois diferentes programas de saúde, é reforçado o fato de que o envelhecimento causaria uma diminuição da qualidade de vida, contudo, os autores apontam que a inatividade física revela-se um importante fator e não simplesmente o fator idade. Já Parahyba, Veras e Melber (2005) ao analisar a incapacidade funcional entre mulheres idosas, a partir da PNAD, indicam que o sedentarismo é um fator de risco, assim como o tabagismo e a má alimentação, os quais podem conduzir a incapacidade funcional.

No grupo estudado, pode-se perceber que existe uma relação intrínseca entre os diferentes domínios da qualidade de vida, apesar de terem diferenças nas suas avaliações (conforme as médias dos mesmos). Trentini et al. (2010), através de um estudo para identificar as variáveis relevantes, associadas com a qualidade de vida de adultos idosos, encontraram que em idosos de classe social mais elevada, a qualidade de vida indicou valores mais elevados. Outro resultado foi que as pessoas mais saudáveis (a partir de sua percepção pessoal) obtiveram as médias mais elevadas em todos os domínios. Tal achado possui similaridade com a amostra deste estudo pela questão da prática de atividade física regular, a qual pode ter influenciado os escores elevados nos domínios do SF-36.

A correlação positiva significativa entre os domínios do SF-36 indica que a qualidade de vida é caracterizada por um conjunto de variáveis interdependentes, as quais se influenciam mutuamente. Deste modo, ao se optar pelos pressupostos teóricos que a orientam, é preciso considerar, em programas de atenção às pessoas, o alcance de objetivos que contemplem essas 
dimensões múltiplas. O que muitas vezes é possível perceber em programas de atenção à saúde, baseados em atividade física para a população idosa, especificamente, é que muitas vezes não são contemplados aspectos que são fundamentais para o seu sucesso. Um dos exemplos mais claros é a necessidade de uma equipe multiprofissional e a facilidade de acesso aos locais das atividades, as quais têm sido enfatizadas como necessárias nos trabalhos propostos em unidades de saúde. Ou seja, é preciso proporcionar encontros semanais regulares de atividade física, em conjunto com um trabalho de promoção de saúde, através da atenção de enfermeiros, psicólogos, médicos, fisioterapeutas, odontologistas, assistentes sociais, entre outros. Desta forma, acredita-se poder atingir uma melhor qualidade de vida, de acordo com sua concepção plena.

Ao relacionar os resultados com outros estudos utilizando o SF-36 é possível perceber que as médias de adultos com idade mais avançada praticantes de atividade física deste estudo são mais elevadas. No estudo de Lemos et al. (2006) com idosos osteoporóticos, as médias em todos os domínios são inferiores, fato destacado pelos autores ao comparar seus resultados também com outras amostras.

Mota et al. (2006) analisaram a relação entre atividade física e qualidade de vida associada à saúde de idosos participantes, e não participantes, em programas regulares de atividade física, sendo que os resultados dos domínios, mesmo no grupo praticante, foram menores que da amostra atual. No estudo de Pimenta et al. (2008) com idosos aposentados, os resultados seguem esse mesmo padrão. Castro et al. (2007) investigaram a influência de programas de intervenção na qualidade de vida de adultos de meia-idade e idosos, sendo que os resultados indicaram uma melhora apenas no domínio EGS do SF-36, não ocorrendo diferença entre os dois grupos de idade. Os resultados indicaram valores similares nos domínios CF e DOR.

A qualidade de vida relacionada à saúde de adultos, com idade entre 50 e 80 anos, praticantes de atividade física regular, mostrou-se com valores superiores, ao ser comparada com outros estudos, a partir do SF-36. Ao mesmo tempo, que ao se referir ao protocolo do estudo em que a maior pontuação em cada domínio é 100, também se percebe valores satisfatórios com no mínimo 70\% de pontuação na média dos domínios. Trentini et al. (2010) aponta como sugestão no seu estudo, a necessidade de analisar diferentes escalas de idade na população idosa, para tentar acompanhar as mudanças decorrentes da idade. Bem como, indicam que seria útil investigar diferentes cenários que promovem a qualidade de vida, tais como a atividade física regular. 
Os sujeitos desta pesquisa são participantes de um projeto de extensão, em média há três anos. O trabalho envolve o desenvolvimento de atividades físicas e de lazer, organizadas de acordo com diferentes componentes da aptidão física, direcionado à saúde (por exemplo: força com oficinas de musculação e ginástica localizada; resistência aeróbica, através de hidroginástica e jogging aquático; flexibilidade, através de alongamento; coordenação e ritmo, através de dança) e de acordo com interesses do lazer (físico, com exercícios físicos; social, com atividades de integração, como bailes e almoços; desenvolvimento, com viagens, coral, percussão, oficina literária). Organizado de acordo com esses pilares, são ofertadas oficinas de cada uma das atividades físicas em quatro turmas, com duas aulas semanais cada, visando favorecer a participação no maior número de atividades. Já as atividades de lazer ocorrem semanalmente, ou mensalmente, e são propostas pelos próprios idosos. Deste modo, os resultados são cumulativos para a maioria da amostra ao longo de todos esses anos, parecendo ser um diferencial, porque nos outros estudos, quando ocorria algum tipo de atividade física a mesma era recente para o grupo (não passando de um ano). Nesta análise, não foi realizada uma comparação entre os sujeitos e a pontuação do SF-36, a partir do tempo de participação no projeto, a qual será realizada nas próximas publicações, com uma amostragem maior.

A premissa deste estudo foi avaliar a qualidade de vida de adultos entre 50 e 80 anos, praticantes de atividade física regular, utilizando o instrumento SF-36. Os resultados indicaram um nível satisfatório de qualidade de vida, além de interação entre os domínios.

A compreensão dos diferentes domínios da qualidade de vida precisa estar relacionada com outras variáveis, como as características socioeconômicas, as quais não foram objetivo deste estudo. A variável analisada como tendo influência na qualidade de vida, nesta pesquisa, foi a prática regular de atividade física. Os resultados mostram bons índices, de acordo com o protocolo do estudo e ao compararem-se esses com outros grupos, também se verificou valores superiores na HRQL.

A diferença de pontuação entre os domínios do SF-36 pode nortear o desenvolvimento de novos objetivos para o grupo estudado, porém, reforça a interface entre os mesmos. É conveniente destacar a importância desse tipo de avaliação, além de outras possíveis, em grupos de idosos praticantes de atividade física regular. 


\title{
abstract
}

The health-related quality of life - HRQOL is a multidimensional issue with integration of physical health, psychological well-being and social satisfaction factors. The aim of this study was to evaluate the health-related quality of life - HRQOL in ageing people who practice regular physical activity. This study was a descriptive cross-sectional and the sample included 40 adults, aged between 50 and 80 years, attending a University Extension Program. The instrument used was the Brazilian version of Short Form-36. Descriptive statistical analysis was used to summarize HRQL data and Pearson's correlation for comparison between domains. The score of the domains in descending order were: AS, AF, AE, EGS, CF, SM, VIT, DOR. There was a significant positive correlation between the majority of SF-36. The correlations were not significant between EGS $\times$ AS, SM $\times$ AF. The age was correlated only with the EGS field. The HRQL of the sample proved to be higher, when compared with other studies. The values reached a minimum score of $70 \%$ in average scores. The results indicated a satisfactory level of HRQOL and a significant relationship between different aspects of health-related quality of life.

keywords

Aging. Quality of Life. Physical Activity. Health.

\author{
referências
}

\begin{abstract}
BERLEZI, Evelise Morais; ROSA, Patrícia Viana da; SOUZA, Antônio Carlos Araújo de; SCHNEIDER, Rodolfo Heberto. Comparação antropométrica e do nível de aptidão física de mulheres acima de 60 anos praticantes de atividade física regular e não praticantes. Revista Brasileira de Geriatria e Gerontologia, Rio de Janeiro, v. 9, n. 3, p. 1-13, 2006. Disponível em: <http://unati.uerj.br/tse/scielo.php?script=sci_arttext\&pid=\$18099823 $2006000300005 \& \operatorname{lng}=p t \& n r m=i s o>$. Acesso em: 01 de mar. 2010.
\end{abstract}

CASTRO, Paulo Costa; TAHARA, Norma; REBELATTO, José Rubens; DRIUSSO, Patricia; AVEIRO, Maria Chaves; OISHI, Jorge. Influência da universidade aberta da terceira idade (UATI) e do programa de revitalização (REVT) sobre a qualidade de vida de adultos de meia-idade e idosos. Revista Brasileira de Fisioterapia, São Carlos, v. 11, n. 6, p. 461467, nov./dez., 2007.

CICONELLI, Rozana Mesquita. Tradução para o português e validação do questionário genérico de avaliação da qualidade de vida "Medical Outcomes Study 36-item Short-Form 
Health Survey (SF-36)". 145 p. 1997. Tese (Doutorado em Medicina) - Escola Paulista de Medicina, Universidade de São Paulo, São Paulo, 1997.

FIEDLER, Mariarosa Mendes; PERES, Karen Glazer. Capacidade funcional e fatores associados em idosos do Sul do Brasil: um estudo de base populacional. Caderno de Saúde Pública, Rio de Janeiro, v. 24, n. 2, p. 409-415, 2008.

FLECK, Marcelo Pio de Almeida. Problemas conceituais em qualidade de vida. In: FLECK, Marcelo Pio de Almeida. A avaliação da qualidade de vida. Porto Alegre: Artmed, 2008. p. 19-28.

GONÇALVES, Andréa Kruger. Ser idoso no mundo: o indivíduo idoso e a vivência de atividades físicas como meio de afirmação e identidade social. 230p. 1999. Tese (Doutorado em Psicologia) - Instituto de Psicologia, Universidade de São Paulo, São Paulo, 1999.

GONÇALVES, Andréa Kruger; GROENWALD, Rosa Maria Freitas. Qualidade de vida e estilo de vida ativo no envelhecimento. Porto Alegre: Novo Tempo, 2005.

LEMOS, Maria Carolina Davel; MIYAMOTO, Samira Tatiyama; VALIM, Valéria; NATOUR, Jamil. Qualidade de Vida em Pacientes com Osteoporose: Correlação entre OPAQ e SF-36. Revista Brasileira de Reumatologia, São Paulo, v. 46, n. 5, p. 323-328, set./out., 2006.

LIMA; Margarete Guimarães; BARROS, Marilisia Berti de Azevedo; GALVÃO CÉSAR, Chester Luiz; GOLDBAUM, Moisés; CARANDINA, Luana; CICONELLI, Rozana Mesquita. Health related quality of life among the elderly: a population-based study using SF-36 survey. Cademo de Saúde Pública, Rio de Janeiro, v. 25, n. 10, p. 2159-2167, out., 2009 .

MOTA, Jorge; RIBEIRO José Luis; CARVALHO, Joana; MATOS, Margarida Gaspar de. Atividade física e qualidade de vida associada à saúde em idosos participantes e não participantes em programas regulares de atividade física. Revista Brasileira de Educação Física e Esporte, São Paulo, v. 20, n. 3, p. 219-25, jul./set., 2006.

NAVEGA, Marcelo Tavella; OISHI, Jorge. Comparação da qualidade de vida relacionada à saúde entre mulheres na pós-menopausa praticantes de atividade física com e sem osteoporose. Revista Brasileira de Reumatologia, São Paulo, v. 47, n. 4, p. 258-264, jul. ago., 2007.

NERI, Anita Liberalesso (Org.). Psicologia do envelhecimento. Campinas: Papirus, 1995.

PARAHYBA, Maria Isabel; VERAS, Renato; MELBER, David. Incapacidade funcional entre as mulheres idosas no Brasil. Revista de Saúde Pública, São Paulo, v. 39, n. 3, p. 383-391, 2005.

PETERSON, Matthew J.; GIULIANI, Carol; MOREY, Míriam C.; PIEPER, Carl F.; EVENSON, Kelly R.; MERCER, Vicki; COHEN, Harvey; VISSER, Marjolein; BRACH, Jennifer; KRITCHEVSKY, Stephen B.; GOODPASTER, Bret H.; RUBIN, Susan; ELD, Suzanne S.; NEWMAN, Anne B.; SIMONSICK, Eleanor M. Physical activity as a preventative factor for frailty: the health, aging, and body composition study. Joumal of Gerontology: Medical Sciences, Oxford, v. 64A, n. 1, p. 61-68, jan., 2009. Disponível em: <http://biomedgerontology.oxfordjournals.org/cgi/reprint/64A/1/61>. Acesso em: 22 de maio 2010

PIMENTA, Fausto Alozio Pedrosa; SIMIL, Fabrícia Fonseca; TÔRRES, Henrique Osvaldo Gama; AMARAL, Carlos Faria Santos; REZENDE, Camila Farnese; COELHO, Thaissa Oliveira; REZENDE, Nilton Alves de. Avaliação da qualidade de vida de aposentados com a utilização do questionário SF-36. Revista da Associação de Médica Brasileira, São Paulo, v. 54, n. 1, p. 55-60, 2008.

PU, Charles T.; NELSON, Miriam E. Exercício físico e envelhecimento. In: FRONTERA, Walter R.; DAWSON, David M.; SLOVIK, David M. Exercicio Físico e Reabilitação. Porto Alegre: Artmed, 2001. p. 347-372. 
Recebido: 23/12/2010

1ª Revisão: 14/03/2011

2a Revisão: 17/05/2011

3a Revisão: 19/07/2011

Aceite Final: 03/08/2011

PUGGAARD, Lis. Effects of training on functional performance in 65, 75 and 85 yearold women: experiences derived from community based studies in Odense, Denmark. Scandinavian Joumal of Medicine \& Science in Sponts, v. 13, n. 1, p. 70-76, 2003

REBELATTO, José Rubens; CALVO, José Ignacio; OREJUELA, J.R.; PORTILLO, J.C. Influência de um programa de atividade física de longa duração sobre a força muscular manual e a flexibilidade corporal de mulheres idosas. Revista Brasileira de Fisioterapia, São Carlos, v. 10, n. 1, p. 127-132, 2006. Disponível em: <http://doi:10.1590/S141335552006000100017>. Acesso em: 18 de abr. 2010.

SGUIZZATTO, Guilherme Turolla; GARCEZ-LEME, Luiz Eugênio; CASIMIRO, Luciana. Evaluation of the quality of life among elderly female athletes. São Paulo Medical Joumal, São Paulo, v. 124, n. 5, p. 304-305, 2006.

SOUZA, Liliana; GALANTE, Helena; FIGUEIREDO, Daniela. Qualidade de vida e bemestar dos idosos: um estudo exploratório na população portuguesa. Revista de Saúde Pública, São Paulo, v. 37, n. 3, 2003, p. 364-371.

SPIRDUSO, Waneen. Dimensões físicas do envelhecimento. Barueri: Manole, 2005.

TRENTINI, Clarissa Marceli; CHACHAMOVICH, Eduardo; WAGNER, Gabriela Peretti; MÜLLER, Daniela Helena; HIRAKATA, Vânia Naomi; FLECK, Marcelo Pio de Almeida. Quality of life (QoL) in a brazilian sample of older adults: the role of sociodemographic variables and depression symptoms. Applied Research Quality Life, v. 5, p. 165-162, 2010. 